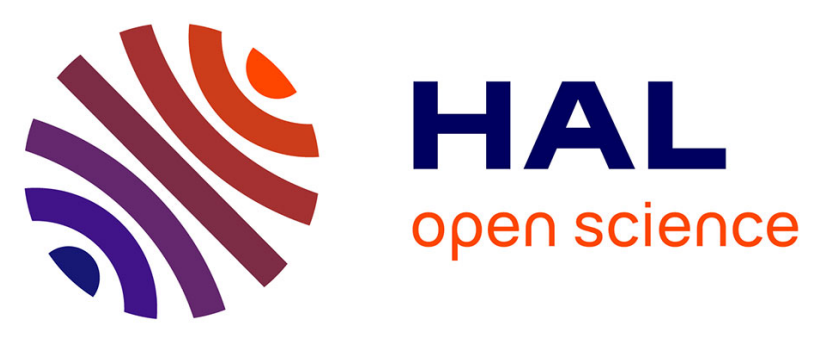

\title{
Models defined in stratified-plate domains. A review on efficient 3D PGD-based solution procedures
}

\author{
Brice Bognet, Chady Ghnatios, Remy Lurot, Anaïs Barasinski, Adrien
}

Leygue, Christophe Binetruy, Francisco Chinesta

\section{- To cite this version:}

Brice Bognet, Chady Ghnatios, Remy Lurot, Anaïs Barasinski, Adrien Leygue, et al.. Models defined in stratified-plate domains. A review on efficient 3D PGD-based solution procedures. Revue des composites et des matériaux avancés = Journal of Composite and Advanced Materials, 2014, 24 (1), pp.97-109. 10.3166/RCMA.24.97-109 . hal-01711391

\author{
HAL Id: hal-01711391 \\ https://hal.science/hal-01711391
}

Submitted on 17 Feb 2018

HAL is a multi-disciplinary open access archive for the deposit and dissemination of scientific research documents, whether they are published or not. The documents may come from teaching and research institutions in France or abroad, or from public or private research centers.
L'archive ouverte pluridisciplinaire $\mathbf{H A L}$, est destinée au dépôt et à la diffusion de documents scientifiques de niveau recherche, publiés ou non, émanant des établissements d'enseignement et de recherche français ou étrangers, des laboratoires publics ou privés. 


\title{
Models defined in stratified-plate domains
}

\author{
A review on efficient 3D PGD-based solution \\ procedures
}

\author{
Brice Bognet ${ }^{1}$, Chady Ghnatios ${ }^{1}$, Remy Lurot ${ }^{1,2}$, \\ Anais Barasinski ${ }^{1}$, Adrien Leygue ${ }^{1}$, \\ Christophe Binetruy ${ }^{1}$, Francisco Chinesta ${ }^{1}$
}

1. GEM, UMR CNRS - Ecole Centrale de Nantes, 1 rue de la Noe, BP 92101, F-44321 Nantes cedex 3, France

\{Brice.Bognet,Chady.Ghnatios, Remy.Lurot,Anais.Barasinski,Adrien.Leygue

,Christophe.Binetruy,Francisco.Chinesta\}@ec-nantes.fr

2. IRT Jules Verne, Chemin du Chaffault, F- 44340 Bougenais, France

\begin{abstract}
The solution of $3 D$ models in degenerated geometries in which some characteristic dimensions are much lower than the other ones - e.g. beams, plates or shells - is a tricky issue when using standard mesh-based discretization techniques. Separated representations allow decoupling the meshes used for approximating the solution along each coordinate. Thus, in plate or shell geometries $3 D$ solutions can be obtained from a sequence of $2 D$ and $1 D$ problems allowing a fine and accurate representation of the solution evolution along the thickness coordinate while keeping the computational complexity characteristic of $2 D$ simulations. In this work we revisit the application of such methodology for addressing different physics (thermal models, solid and fluid mechanics and electromagnetic problems) in such degenerated geometries.

RÉSUMÉ. La résolution de problèmes $3 D$ sur des domaines qui présentent un important rapport d'aspect tels que les structures minces (plaques et coques) représente un défi pour les méthodes de résolution utilisant un maillage. Une représentation séparée permet de découpler les maillages utilisés pour approximer la solution selon les différentes coordonnées. Ainsi, la solution 3D d'un problème défini sur une géométrie plaque ou coque peut être obtenue par la résolution d'une séquence de problèmes $1 D$ et 2D. Cette représentation permet d'obtenir une solution précise sur l'ensemble du domaine (en particulier dans la direction de l'épaisseur, maillée en 1D) en utilisant des maillages adaptés suivant les différentes dimensions, ceci en gardant une complexité 2D. Cette méthode est appliquée ici pour un certain nombre de physiques (thermique,
\end{abstract}


mécanique des solides et des fluides, électromagnétisme) définies sur des domaines à fort rapport d'aspect.

KEYWORDS: plate domains, shell domains, laminates, PGD, model order reduction. MOTS-CLÉS : plaques, coques, stratifiés, PGD, réduction de modèles.

\section{Introduction}

Plates and shells are very common in nature and thus they inspired engineers that used both from the very beginning of structural mechanics. In general the design of such structural elements requires the calculation of stresses, strains and displacements for the design loads. Strains and stresses are related by the so-called constitutive law, the simplest one being the linear elasticity. Moreover other physics can be encountered in these structural elements as for example thermal processes, electromagnetism or fluid flows. Typically composites manufacturing processes usually involve a resin flowing into the double scale porous media, that polymerize by a thermal, chemical or electromagnetic activation. Welding processes could also involve the use of thermal, mechanical or electromagnetic devices.

Thus, structural or processes design always involve the solution of a set of partial differential equations in the degenerate domain of the plate or the shell with appropriate initial and boundary conditions. These domains are degenerated because one of its characteristic dimensions (the thickness in the present case) is much lower that the other characteristic dimensions. We will understand the consequences of such degeneracy later. When analytical solutions are neither available nor possible because the geometrical or behavior complexities, the solution must be calculated by invoking any of the available numerical techniques (finite elements, finite differences, finite volumes, methods of particles, ...).

In the numerical framework the solution will be only obtained in a discrete number of points, usually called nodes, properly distributed in the domain. From the solution at those points, it can be interpolated at any other point in the domain. In general regular nodal distributions are preferred because they offer the best accuracy. In the case of degenerated plate or shell domains one could expect that if the solution evolves significantly in the thickness direction, a large enough number of nodes must be distributed along the thickness direction to ensure the accurate representation of the field evolution in that direction. In that case, a regular nodal distribution in the whole domain will imply the use of an extremely large number of nodes with the consequent impact on the numerical solution efficiently.

When simple behaviors and domains were considered, plate and shell theories were developed allowing, through the introduction of some hypotheses, reducing the $3 \mathrm{D}$ complexity to the $2 \mathrm{D}$ related to the problem now formulated by considering the in-plane coordinates. The use of these theories was extended gradually for addressing 
larger and more complex geometries (anisotropic laminates, ... ) and behaviors (Timoshenko and Woinowsky-Krieger 1959; Zhang and Yang 2009; Qatu 2012). These simplified "plate models" exist for most of the physics just referred, but their applicability is in many cases too constrained.

Moreover, as soon as richer physics are involved in the models and the considered geometries differ of those ensuring the validity of the different reduction hypotheses, efficient simulations are compromised. For example in composites manufacturing processes of large parts many reactions and thermal processes inducing significant evolutions on the thermomechanical fields in the thickness occur. These inhomogeneities are at the origin of residual stresses and the associated distortion of the formed parts.

In these circumstances as just indicated the reduction from the $3 \mathrm{D}$ model to a $2 \mathrm{D}$ simplified one is not obvious, and 3D simulations appear many times as the only valid route for addressing such models, that despite the fact of being defined in degenerated geometries (plate or shell) they seem requiring a fully 3D solution. In order to integrate such calculations (fully 3D and implying an impressive number of degrees of freedom) in usual design procedures, a new efficient (fast and accurate) solution procedure is needed.

A new discretization technique based on the use of separated representations was recently proposed for addressing space-time nonlinear models (Ladeveze, 1999) and then it was generalized for defining general separated representations of solutions involving conformational coordinates (Ammar et al., 2006), space and time and even parameters considered as extra-coordinates. The interested reader can refer to the recent reviews (Chinesta et al., 2010; Chinesta, Ammar et al., 2011; Chinesta, Ladeveze and Cueto, 2011) and the references therein.

A direct consequence was the separated representations involving the space coordinates. Thus an in-plane-out-of-plane decomposition was proposed for solving flow problems in laminates (Chinesta, Ammar et al., 2011; Ghnatios et al., 2013), thermal problems in extruded geometries (Leygue et al., 2013) and laminates (Chinesta et al., 2013), elasticity problems in plates (Bognet et al., 2012) and shells geometries (Bognet et al., 2013). In general the 3D solution was obtained from the solution of a sequence of $2 \mathrm{D}$ problems (the ones involving the in-plane coordinates) and 1D problems (the ones involving the coordinate related to the plate thickness).

It is important emphasizing the fact that these approaches are radically different to standard plate and shell approaches. We proposed a 3D solver able to compute the different unknown fields without the necessity of introducing any hypothesis. The most outstanding advantage is that $3 \mathrm{D}$ solutions can be obtained with a computational cost characteristic of standard 2D solutions.

In this work we revisit the in-plane-out-of-plane representation and its application for addressing different physics (thermal, elastic, flow and electromagnetic models) in plate domains, eventually consisting of a laminate composed of several anisotropic plies. 


\section{Models defined in plate and shell geometries}

\subsection{Elastic problem in plate domains}

We proposed in Bognet et al. (2012) and original in-plane-out-of-plane decomposition of the 3D elastic solution in a plate geometry. The elastic problem was defined in a plate domain $\Xi=\Omega \times \mathcal{I}$ with $\mathbf{x}=\left(x_{1}, x_{2}\right) \in \Omega, \Omega \subset \mathcal{R}^{2}$ and $x_{3} \in \mathcal{I}$, $\mathcal{I}=[0, H] \subset \mathcal{R}$, being $H$ the plate thickness. The separated representation of the displacement field $\mathbf{u}=\left(u_{1}, u_{2}, u_{3}\right)$ reads:

$$
\mathbf{u}\left(x_{1}, x_{2}, x_{3}\right)=\left(\begin{array}{c}
u_{1}\left(x_{1}, x_{2}, x_{3}\right) \\
u_{2}\left(x_{1}, x_{2}, x_{3}\right) \\
u_{3}\left(x_{1}, x_{2}, x_{3}\right)
\end{array}\right) \approx \sum_{i=1}^{N}\left(\begin{array}{c}
P_{1}^{i}\left(x_{1}, x_{2}\right) \cdot T_{1}^{i}\left(x_{3}\right) \\
P_{2}^{i}\left(x_{1}, x_{2}\right) \cdot T_{2}^{i}\left(x_{3}\right) \\
P_{3}^{i}\left(x_{1}, x_{2}\right) \cdot T_{3}^{i}\left(x_{3}\right)
\end{array}\right)
$$

where $P_{k}^{i}, k=1,2,3$, are functions of the in-plane coordinates $\mathbf{x}=\left(x_{1}, x_{2}\right)$ whereas $T_{k}^{i}, k=1,2,3$, are functions involving the thickness coordinate $x_{3}$.

Expression (1) can be written in a more compact form by using the Hadamard product:

$$
\mathbf{u}\left(\mathbf{x}, x_{3}\right) \approx \sum_{i=1}^{N} \mathbf{P}^{i}(\mathbf{x}) \circ \mathbf{T}^{i}\left(x_{3}\right)
$$

where vectors $\mathbf{P}^{i}$ and $\mathbf{T}^{i}$ contains functions $P_{k}^{i}$ and $T_{k}^{i}$ respectively.

Because neither the number of terms in the separated representation of the displacement field nor the dependence on $x_{3}$ of functions $T_{k}^{i}$ are assumed a priori, the approximation is flexible enough for representing the fully 3D solution, being obviously more general than theories assuming particular a priori evolutions in the thickness direction $x_{3}$.

Let's consider a linear elasticity problem on a plate domain $\Xi=\Omega \times \mathcal{I}$. The weak formulation reads:

$$
\int_{\Xi} \boldsymbol{\epsilon}\left(\mathbf{u}^{*}\right)^{T} \cdot \mathbf{K} \cdot \boldsymbol{\epsilon}(\mathbf{u}) d \mathbf{x}=\int_{\Xi} \mathbf{u}^{*} \cdot \mathbf{f}_{d} d \mathbf{x}+\int_{\Gamma_{N}} \mathbf{u}^{*} \cdot \mathbf{F}_{d} d \mathbf{x}
$$

with $\mathbf{K}$ the generalized $6 \times 6$ Hooke tensor, $\mathbf{f}_{d}$ represents the volumetric body forces while $\mathbf{F}_{d}$ represents the traction applied on the boundary $\Gamma_{N}$. In what follows we assume that $\mathbf{K}, \mathbf{f}_{d}$ and $\mathbf{F}_{d}$ accepts an in-plane-out-of-plane separated representation (we come back to this issue later).

The separated representation constructor proceeds by computing a term of the sum at each iteration. Assuming that the first $n-1$ modes (terms of the finite sum) of the solution were already computed, $\mathbf{u}^{n-1}\left(\mathbf{x}, x_{3}\right)$ with $n \geq 1$, the solution enrichment reads:

$$
\mathbf{u}^{n}\left(\mathbf{x}, x_{3}\right)=\mathbf{u}^{n-1}\left(\mathbf{x}, x_{3}\right)+\mathbf{P}^{n}(\mathbf{x}) \circ \mathbf{T}^{n}\left(x_{3}\right)
$$

where both vectors $\mathbf{P}^{n}$ and $\mathbf{T}^{n}$ containing functions $P_{i}^{n}$ and $T_{i}^{n}(i=1,2,3)$ depending on $\mathbf{x}$ and $x_{3}$ respectively, are unknown at the present iteration, resulting in a non-linear problem. 
We proceed by considering the simplest linearization strategy, an alternated directions fixed point algorithm, that proceeds by calculating $\mathbf{P}^{n}$ by assuming $\mathbf{T}^{n}$ known, and then by updating $\mathbf{T}^{n}$ from the just calculated $\mathbf{P}^{n}$. The iteration procedure continues until convergence, that is, until reaching the fixed point.

When $\mathbf{T}^{n}$ is assumed known, we consider the test function $\mathbf{u}^{\star}$ given by $\mathbf{P}^{\star} \circ \mathbf{T}^{n}$. By introducing the trial and test functions into the weak form and then integrating in $\mathcal{I}$ because all the functions depending on the thickness coordinate are known, we obtain a $2 \mathrm{D}$ weak formulation defined in $\Omega$ whose discretization (by using a standard discretization strategy, e.g. finite elements) allows computing $\mathbf{P}^{n}$.

Analogously, when $\mathbf{P}^{n}$ is assumed known, the test function $\mathbf{u}^{\star}$ is given by $\mathbf{P}^{n} \circ \mathbf{T}^{\star}$. By introducing the trial and test functions into the weak form and then integrating in $\Omega$ because all the functions depending on the in-plane coordinates $\mathrm{x}$ are at present known, we obtain a 1D weak formulation defined in $\mathcal{I}$ whose discretization (using any technique for solving standard ODE equations) allows computing $\mathbf{T}^{n}$.

As discussed in Bognet et al. (2012) this separated representation allows computing 3D solutions while keeping a computational complexity characteristic of $2 \mathrm{D}$ solution procedures. If we consider a hexahedral domain discretized using a regular structured grid with $N_{1}, N_{2}$ and $N_{3}$ nodes in the $x_{1}, x_{2}$ and $x_{3}$ directions respectively, usual mesh-based discretization strategies imply a challenging issue because the number of nodes involved in the model scales with $N_{1} \cdot N_{2} \cdot N_{3}$, however, by using the separated representation and assuming that the solution involves $N$ modes, one must solve about $N$ 2D problems related to the functions involving the in-plane coordinates $\mathbf{x}$ and the same number of $1 \mathrm{D}$ problems related to the functions involving the thickness coordinate $x_{3}$. The computing time related to the solution of the onedimensional problems can be neglected with respect to the one required for solving the two-dimensional ones. Thus, the resulting complexity scales as $N \cdot N_{1} \cdot N_{2}$. By comparing both complexities we can notice that as soon as $N_{3} \gg N$ the use of separated representations leads to impressive computing time savings, making possible the solution of models never until now solved, and even using light computing platforms (Bognet et al., 2012).

\subsection{Elastic problem in shell domains}

The shell domain $\Omega^{S}$, assumed with constant thickness, can be described from a reference surface $\mathbf{X}$, that in what follows will be identified to the shell middle surface but that in the general case could be any other one, parametrized by the coordinates $\xi, \eta$, that is $\mathbf{X}(\xi, \eta)$, where:

$$
\mathbf{X}(\xi, \eta)=\left(\begin{array}{c}
X_{1}(\xi, \eta) \\
X_{2}(\xi, \eta) \\
X_{3}(\xi, \eta)
\end{array}\right)
$$


Being $\mathbf{n}$ the unit vector normal to the middle surface, the shell domain $\Omega^{S}$ can be parametrized from:

$$
\mathbf{x}(\xi, \eta, \zeta)=\mathbf{X}(\xi, \eta)+\zeta \cdot \mathbf{n}
$$

The geometrical transformation $(\xi, \eta, \zeta) \rightarrow\left(x_{1}, x_{2}, x_{3}\right)$ involves

$$
\tilde{\mathbf{F}}=\left[\frac{\partial \mathbf{x}}{\partial \xi} \frac{\partial \mathbf{x}}{\partial \eta} \mathbf{n}\right]
$$

With the weak form of the elastic problem defined in the reference domain $\Xi=$ $\Omega \times \mathcal{I}$, with $(\xi, \eta) \in \Omega$ and $\zeta \in \mathcal{I}$, the situation is quite similar to the one encountered in the analysis of elastic problems in plate geometries, that was addressed in Bognet et al. (2012).

In Bognet et al. (2013) we considered the in-plane-out-of-plane separated representation of the displacement field, similar to (1) but now involving the coordinates $(\xi, \eta, \zeta)$

$$
\mathbf{u}(\xi, \eta, \zeta)=\left(\begin{array}{c}
u_{1}(\xi, \eta, \zeta) \\
u_{2}(\xi, \eta, \zeta) \\
u_{3}(\xi, \eta, \zeta)
\end{array}\right) \approx \sum_{i=1}^{N}\left(\begin{array}{c}
P_{1}^{i}(\xi, \eta) \cdot T_{1}^{i}(\zeta) \\
P_{2}^{i}(\xi, \eta) \cdot T_{2}^{i}(\zeta) \\
P_{3}^{i}(\xi, \eta) \cdot T_{3}^{i}(\zeta)
\end{array}\right)
$$

or in a more compact form

$$
\mathbf{u}(\xi, \eta, \zeta) \approx \sum_{i=1}^{N} \mathbf{P}^{i}(\xi, \eta) \circ \mathbf{T}^{i}(\zeta)
$$

\subsection{Darcy's flow model}

We now illustrate the application of separated representations to the modeling of resin transfer molding processes deeply addressed in Chinesta, Ammar et al. (2011). We consider the flow within a porous medium in a plate domain $\Xi=\Omega \times \mathcal{I}$ with $\Omega \subset \mathcal{R}^{2}$ and $\mathcal{I}=[0, H] \subset \mathcal{R}$. The governing equation is obtained by combining Darcy's law, which relates the fluid velocity to the pressure gradient

$$
\mathbf{v}=-\mathbf{K} \cdot \nabla p
$$

and the incompressibility constraint,

$$
\nabla \cdot \mathbf{v}=0
$$

Introduction of Eq. (10) into Eq. (11) yields a single equation for the pressure field

$$
\nabla \cdot(\mathbf{K} \cdot \nabla p)=0
$$

Remark: The heat equation being formally similar to Eq. (12) the considerations that follow also apply for the solution of thermal models in plate geometries. 
Again we assume the permeability separability

$$
\mathbf{K}(x, y, z)=\sum_{i=1}^{P} \mathbf{K}_{i}(\mathbf{x}) \cdot \xi_{i}(z)
$$

where $\mathbf{x}$ denotes the in-plane coordinates, i.e. $\mathbf{x}=(x, y) \in \Omega$.

The weak form of Eq. (12) reads:

$$
\int_{\Xi} \nabla p^{*} \cdot(\mathbf{K} \cdot \nabla p) d \Xi=0
$$

for all test functions $p^{*}$ selected in an appropriate functional space. Dirichlet boundary conditions are imposed for the pressure at the inlet and outlet of the flow domain, while zero flux (i.e. no flow) is imposed elsewhere as a weak boundary condition. We seek an approximate solution $p(x, y, z)$ in the separated form:

$$
p(\mathbf{x}, z) \approx \sum_{j=1}^{N} X_{j}(\mathbf{x}) \cdot Z_{j}(z)
$$

The PGD algorithm then proceeds as follows. Assume that the first $n$ functional products have been computed, i.e.

$$
p^{n}(\mathbf{x}, z)=\sum_{j=1}^{n} X_{j}(\mathbf{x}) \cdot Z_{j}(z)
$$

is a known quantity. We must now perform an enrichment step to obtain

$$
p^{n+1}(\mathbf{x}, z)=p^{n}(\mathbf{x}, z)+R(\mathbf{x}) \cdot S(z)
$$

The test function involved in the weak form is given by

$$
p^{*}(\mathbf{x}, z)=R^{*}(\mathbf{x}) \cdot S(z)+R(\mathbf{x}) \cdot S^{*}(z)
$$

Introducing Eqs. (17) and (18) into Eq. (14), we obtain

$$
\begin{gathered}
\int_{\Xi}\left(\left(\begin{array}{c}
\tilde{\nabla} R^{*} \cdot S \\
R^{*} \cdot \frac{d S}{d z}
\end{array}\right)+\left(\begin{array}{c}
\tilde{\nabla} R \cdot S^{*} \\
R \cdot \frac{d S^{*}}{d z}
\end{array}\right)\right) \cdot\left(\mathbf{K} \cdot\left(\begin{array}{c}
\tilde{\nabla} R \cdot S \\
R \cdot \frac{d S}{d z}
\end{array}\right)\right) d \Xi= \\
=-\int_{\Xi}\left(\left(\begin{array}{c}
\tilde{\nabla} R^{*} \cdot S \\
R^{*} \cdot \frac{d S}{d z}
\end{array}\right)+\left(\begin{array}{c}
\tilde{\nabla} R \cdot S^{*} \\
R \cdot \frac{d S^{*}}{d z}
\end{array}\right)\right) \cdot \mathbf{Q}^{n} d \Xi
\end{gathered}
$$

where $\tilde{\nabla}$ denotes the plane component of the gradient operator, i.e. $\tilde{\nabla}^{T}=\left(\frac{\partial}{\partial x}, \frac{\partial}{\partial y}\right)$ and $\mathbf{Q}^{n}$ is a flux term known at step $n$ :

$$
\mathbf{Q}^{n}=\mathbf{K} \cdot \sum_{j=1}^{n}\left(\begin{array}{c}
\tilde{\nabla} X_{j}(\mathbf{x}) \cdot Z_{j}(z) \\
X_{j}(\mathbf{x}) \cdot \frac{d Z_{j}(z)}{d z}
\end{array}\right)
$$


As discussed previously, each enrichment step of the PGD algorithm is a nonlinear problem which must be performed by means of a suitable iterative process. The simplest one proceeds assuming $R(\mathbf{x})$ known to obtain $S(z)$, and then updating $R(\mathbf{x})$. The process continues until reaching convergence. The converged solutions provide the next functional product of the PGD: $R(\mathbf{x}) \rightarrow X_{n+1}(\mathbf{x})$ and $S(z) \rightarrow Z_{n+1}(z)$.

\subsection{Stokes flow model}

The Stokes model is defined in $\Xi=\Omega \times \mathcal{I}$ and reads for an incompressible fluid:

$$
\left\{\begin{array}{l}
\nabla p=\nabla \cdot(\eta \cdot \nabla \mathbf{v}) \\
\nabla \cdot \mathbf{v}=0
\end{array}\right.
$$

To circumvent the issue related to stable mixed formulations (LBB conditions) within the separated representation we consider a penalty formulation that modifies the mass balance by introducing a penalty coefficient $\lambda$ small enough

$$
\nabla \cdot \mathbf{v}+\lambda \cdot p=0
$$

or more explicitly

$$
p=-\frac{1}{\lambda}\left(\frac{\partial u}{\partial x}+\frac{\partial v}{\partial y}+\frac{\partial w}{\partial z}\right)=-\frac{\nabla \cdot \mathbf{v}}{\lambda}
$$

By replacing it into the momentum balance (first equation in (21)) we obtain

$$
\nabla(\nabla \cdot \mathbf{v})+\xi \Delta \mathbf{v}=0
$$

with $\xi=\eta \cdot \lambda$.

In the present case it suffices considering the separated representation of the velocity field according to (Ghnatios et al., 2013):

$$
\mathbf{v}=\left(\begin{array}{c}
u \\
v \\
w
\end{array}\right) \approx\left(\begin{array}{c}
\sum_{i=1}^{i=N} X_{i}^{u}(x, y) \cdot Z_{i}^{u}(z) \\
i=N \\
\sum_{i=1}^{i=N} X_{i}^{v}(x, y) \cdot Z_{i}^{v}(z) \\
\sum_{i=1}^{i=N} X_{i}^{w}(x, y) \cdot Z_{i}^{w}(z)
\end{array}\right)
$$

that leads to a separated representation of the strain rate, that introduced into the Stokes problem weak form allows the calculation of functions $X_{i}(x, y)$ by solving the corresponding $2 \mathrm{D}$ problems and functions $Z_{i}(z)$ by solving the associated $1 \mathrm{D}$ problems. Because of the one-dimensional character of problems defined in the laminate thickness we can use extremely fine descriptions along the thickness direction without a significant impact on the computational efficiency. 


\section{Laminates}

In the case of laminates, several plies with different thermomechanical properties (eventually anisotropic) are found through the domain thickness. In the case of addressing the flow in a porous preform we could suppose $P$ different anisotropic plies of thickness $h$, each one characterized by a permeability tensor $\mathbf{K}_{i}(x, y)$ that is assumed constant through the ply thickness. Then, we define a characteristic function

$$
\xi_{i}(z)= \begin{cases}1 & z_{i} \leq z \leq z_{i+1} \\ 0 & \text { otherwise }\end{cases}
$$

where $z_{i}=(i-1) \cdot h$ is the location of ply $i$ in the plate thickness. The laminate's permeability is thus given in separated form

$$
\mathbf{K}(x, y, z)=\sum_{i=1}^{P} \mathbf{K}_{i}(\mathbf{x}) \cdot \xi_{i}(z)
$$

where $\mathbf{x}$ denotes the in-plane coordinates, i.e. $\mathbf{x}=(x, y) \in \Omega$.

\subsection{Brinkmann model}

In composites manufacturing processes resin located among the fibers in the reinforcement layers also flows. Many studies have been reported on the squeeze flow of Newtonian and non-Newtonian fluids between rigid parallel flat bodies with or without wall-slip concerning both experimental and numerical analyses, fundamentals and applications (Gibson and Toll 1999; Engmann et al. 2005).

A usual approach for evaluating the resin flow in such circumstances consists of solving the associated Darcy's model. It is well known that Darcy-Stokes coupling at the interlayers generates numerical instabilities because the localized boundary layers whose accurate description requires very rich representations (very fine meshes along the laminate thickness).

In Ghnatios et al. (2013) we proposed the use of the Brinkman model that allows representing in an unified manner both the Darcy and the Stokes behaviors. In order to avoid numerical inaccuracies we use a very fine representation along the thickness direction and for circumventing the exponential increase in the number of degrees of freedom that such a fine representation would imply when extended to the whole laminate domain, we consider again the in-plane-out-of-plane separated representation previously introduced.

The Brinkman model is defined by:

$$
\nabla p=\mu \cdot \mathbf{K}^{-1} \cdot \mathbf{v}+\eta \cdot \Delta \mathbf{v}
$$

where $\mu$ is the dynamic viscosity, $\mathbf{K}$ the layer permeability tensor and $\eta$ the dynamic effective viscosity. 
In the zones where Stokes model applies (resin layers) we assign a very large isotropic permeability $K=1$ (units in the metric system) whereas in the ones occupied by the reinforcement, the permeability is assumed anisotropic, being several orders of magnitude lower, typically $10^{-8}$. Thus the Darcy's component in Eq. (28) does not perturb the Stokes flow in the resin layers, and it becomes dominant in the reinforcement layers. Additionally by choosing this outstanding difference in permeability, representative of the one observed in liquid Resin Infusion process when highly porous distribution media are used, we also want to give the evidence that this type of problem can be addressed by the proposed approach.

\subsection{On the approximation continuity}

All the just addressed models imply second order derivatives in the space coordinates, and then, after integrating by parts to recover their associated weak forms, only continuous approximations are required for both the trial and the test functions. Thus in general in our numerical applications we considered standard piecewise linear functions for approximating the 2D fields defined in $\Omega$ and the $1 \mathrm{D}$ defined in $\mathcal{I}$. Obviously higher order approximations are possible with the only constraint of ensuring continuity.

Electromagnetic models derived from Maxwell's equations usually involves vector potentials that appears in the weak form affected by the curl operator. Let's $\mathbf{A}=$ $\left(A_{x}, A_{y}, A_{z}\right)$ such a potential. We have

$$
(\nabla \times \mathbf{A})^{T}=\left(\frac{\partial A_{z}}{\partial y}-\frac{\partial A_{y}}{\partial z}, \frac{\partial A_{x}}{\partial z}-\frac{\partial A_{z}}{\partial x}, \frac{\partial A_{y}}{\partial x}-\frac{\partial A_{x}}{\partial y}\right)
$$

that proves that $A_{x}$ must be continuous with respect to the coordinates $y$ and $z$ but should be preferably discontinuous in the $x$ coordinate in order to ensure the transfer conditions from one medium to other. The same reasoning applies for the other components of the vector potential.

If for the sake of clarity we consider in what follows the 2D case defined by the coordinates $(x, z)$ we could approximate $A_{x}$ and $A_{z}$ from

$$
\left\{\begin{aligned}
A_{x} & \approx \sum_{i=1}^{i=N} X_{i}^{x}(x) \cdot Z_{i}^{x}(z) \\
A_{z} & \approx \sum_{i=1}^{i=N} X_{i}^{z}(x) \cdot Z_{i}^{z}(z)
\end{aligned}\right.
$$

where $X_{i}^{x}$ and $Z_{i}^{x}$ are approximated using piecewise constant (discontinuous) and linear (continuous) functions of the $x$ and $z$ coordinates respectively. On the other hand $X_{i}^{z}$ and $Z_{i}^{z}$ are approximated using piecewise linear (continuous) and constant (discontinuous) functions of the $x$ and $z$ coordinates respectively. This simple choice ensure the continuity requirement just specified, and constitutes the simplest gener- 


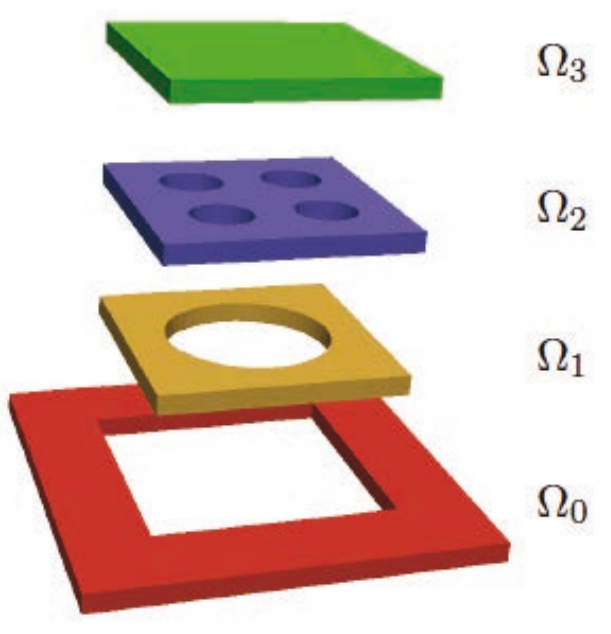

Figure 1. Representation of the domain and the three possible 'patches'

alization of the so-called Nedelec's elements usually considered in electromagnetic numerical simulations.

\section{Parametric models}

The in-plane-out-of-plane separated representation can be enriched by adding model parameters as extra-coordinates and then computing the general parametric solution off-line that could be used in online real time applications as proved in our former papers Bognet et al. (2012) where material and geometrical parameters were included in the parametric solution as extra-coordinates.

In what follows we consider a discrete extra-coordinate to represent different part topologies. The simulation is then performed simultaneously for all possible topologies.

Let us consider the problem defined on the domain $\Omega^{(d)}=\Omega_{0} \cup \Omega_{d}$, where $d \in$ $[1,2,3]$ defined on figure 1 .

The square plate domains $\Omega^{(d)}$ has one (for $d=1$ ), four (for $d=2$ ) or no hole (if $d=3)$. The material is assumed isotropic and homogeneous $(E=150 G P a, \nu=$ $0.3)$. The plate is clamped at one of its lateral faces and a unit shear force applies on the opposite face.

If we consider an arbitrary field $u$, its separated representation reads:

$$
u\left(x_{1}, x_{2}, x_{3}, d\right) \approx \sum_{i=1}^{N} P_{i}\left(x_{1}, x_{2}\right) \cdot T_{i}\left(x_{3}\right) \cdot D_{i}(d)
$$

where $d=1,2,3$ and function $D_{i}(d)=1$ inside the patch $\Omega^{(d)}$ and $D_{i}(d)=0$ elsewhere. The deformed domain is given on figure 2 for each possible scenario. 

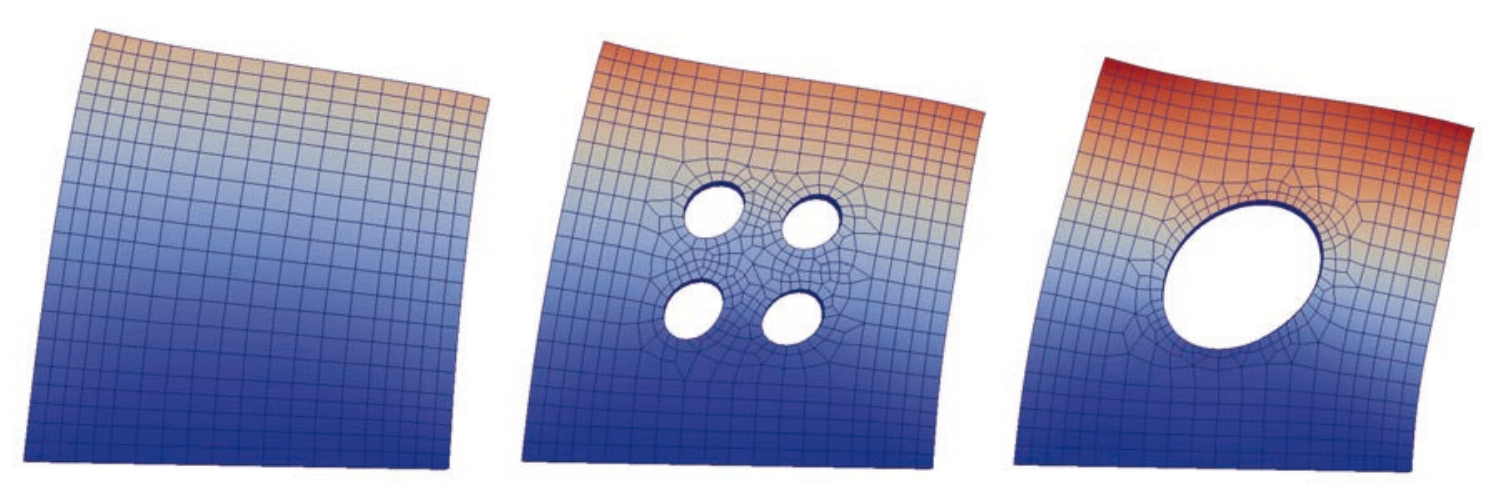

Figure 2. Deformed configuration for (from left to right)

$$
\Omega_{0} \cup \Omega_{1}, \Omega_{0} \cup \Omega_{2} \text { and } \Omega_{0} \cup \Omega_{3}
$$

\section{Conclusions}

In this paper we revisited the use of separated representations for solving models defined in degenerated domains. The issue related to the extremely fine meshes required for capturing rich behaviors along the thickness direction was circumvented by considering in-plane-out-of-plane separated representations of the fields and model parameters involved in the models as well as the geometries in which they are defined.

In the case of quite simple behaviors plate or shell elements were introduced and widely used in many engineering applications. However, in the case of richer behaviors and/or geometries these simplified models fail to describe the more complex behaviors of the associated solutions. Thus for example we proved in Chinesta, Ammar et al. (2011) that when averaging permeability in composite laminates the computed solution differs drastically from the fully 3D solution. The same conclusions apply in the case of thermal models that are formally similar to the ones related to the flow in porous media. We also proved In Bognet et al. (2012) that when geometries become complex the standard elastic plate theories fail for calculating the 3D solution, and in Ghnatios et al. (2013) the necessity of 3D flow calculation was pointed out.

3D solutions as previously discussed in too expensive from a computational point of view, however the used of in-plane-out-of-plane separated representations allows calculating 3D solutions with a computational complexity characteristic of 2D models. In the case of models involving the curl operator we proved that the decomposition also applies and that the approximation can be enhanced by enforcing the approximation discontinuity, by generalizing Nedelec's approximations widely considered in the finite elements framework.

\section{References}

A. Ammar, B. Mokdad, F. Chinesta, R. Keunings. A new family of solvers for some classes of multidimensional partial differential equations encountered in kinetic theory modeling of complex fluids. Journal of Non-Newtonian Fluid Mechanics, 139, 153-176, 2006. 
B. Bognet, A. Leygue, F. Chinesta, A. Poitou, F. Bordeu. Advanced simulation of models defined in plate geometries: 3D solutions with 2D computational complexity. Computer Methods in Applied Mechanics and Engineering, 201, 1-12, 2012.

B. Bognet, A. Leygue, F. Chinesta. Separated representations of 3D elastic solutions in shell geometries. Advanced Modeling and Simulation in Engineering Sciences, 2013. Submitted.

F. Chinesta, A. Ammar, E. Cueto. Recent advances and new challenges in the use of the Proper Generalized Decomposition for solving multidimensional models. Archives of Computational Methods in Engineering, 17/4, 327-350, 2010.

F. Chinesta, A. Ammar, A. Leygue, R. Keunings. An overview of the Proper Generalized Decomposition with applications in computational rheology. Journal of Non Newtonian Fluid Mechanics, 166, 578-592, 2011.

F. Chinesta, P. Ladeveze, E. Cueto. A short review in model order reduction based on Proper Generalized Decomposition. Archives of Computational Methods in Engineering, 18, 395404, 2011.

F. Chinesta, A. Leygue, B. Bognet, Ch. Ghnatios, F. Poulhaon, F. Bordeu, A. Barasinski, A. Poitou, S. Chatel, S. Maison-Le-Poec. First steps towards an advanced simulation of composites manufacturing by Automated Tape Placement. International Journal of Material Forming., 2013. In press DOI: 10.1007/s12289-012-1112-9

J. Engmann, C. Servais, A.S. Burbidge. Squeeze flow theory and applications to rheometry: A review, Journal of Non-Newtonian Fluid Mechanics, 132, 1-27, 2005.

Ch. Ghnatios, F . Chinesta, Ch. Binetruy. 3D Modeling of squeeze flows occurring in composite laminates. International Journal of Material Forming, 1013. Submitted.

A.G. Gibson, S. Toll. Mechanics of the squeeze flow of planar fibre suspensions, Journal of Non-Newtonian Fluid Mechanics, 82/1, 1-24, 1999.

P. Ladevèze. Nonlinear computational structural mechanics, Springer, NY, 1999.

A. Leygue, F. Chinesta, M. Beringhier, T.L. Nguyen, J.C. Grandidier, F. Pasavento, B. Schrefler. Towards a framework for non-linear thermal models in shell domains. International Journal of Numerical Methods for Heat and Fluid Flow, 23/1, 55-73, 2013.

M.S. Qatu. Review of Recent Literature on Static Analyses of Composite Shells: 2000-2010. Open Journal of Composite Materials, 2(3): 61-86, 2012.

S.P. Timoshenko, S.Woinowsky-Krieger. Theory of plates and shells, McGraw-Hill, 1959.

Y.X. Zhang, C.H. Yang. Recent developments in finite element analysis for laminated composite plates. Composite Structures, 88(1):147-157, 2009. 